\title{
Effects of Auraptene on IGF-1 Stimulated Cell Cycle Progression in the Human Breast Cancer Cell Line, MCF-7
}

\author{
Prasad Krishnan $^{1,2}$ and Heather Kleiner-Hancock ${ }^{1}$ \\ ${ }^{1}$ Department of Pharmacology, Toxicology \& Neuroscience, Louisiana State University, Health Sciences Center, \\ 1501 Kings Highway, Shreveport, LA 71130, USA \\ ${ }^{2}$ Department of Veterinary and Biomedical Sciences, Center for Molecular Toxicology and Carcinogenesis, \\ Pennsylvania State University, University Park, PA 16802, USA
}

Correspondence should be addressed to Heather Kleiner-Hancock, hklein@lsuhsc.edu

Received 14 September 2012; Accepted 29 November 2012

Academic Editor: J. Michael Mathis

Copyright () 2012 P. Krishnan and H. Kleiner-Hancock. This is an open access article distributed under the Creative Commons Attribution License, which permits unrestricted use, distribution, and reproduction in any medium, provided the original work is properly cited.

\begin{abstract}
Auraptene is being investigated for its chemopreventive effects in many models of cancer including skin, colon, prostate, and breast. Many mechanisms of action including anti-inflammatory, antiproliferative, and antiapoptotic effects are being suggested for the chemopreventive properties of auraptene. We have previously shown in the $N$-methylnitrosourea induced mammary carcinogenesis model that dietary auraptene $(500 \mathrm{ppm})$ significantly delayed tumor latency. The delay in time to tumor corresponded with a significant reduction in cyclin D1 protein expression in the tumors. Since cyclin D1 is a major regulator of cell cycle, we further studied the effects of auraptene on cell cycle and the genes related to cell cycle in MCF-7 cells. Here we show that auraptene significantly inhibited IGF-1 stimulated S phase of cell cycle in MCF-7 cells and significantly changed the transcription of many genes involved in cell cycle.
\end{abstract}

\section{Introduction}

Breast cancer has been one of the most common cancers among women in the United States. The estimates for 2012 are 226,870 new cases of breast cancer and 39,510 deaths due to breast cancer in women in the United States [1]. In addition to the incidence and death in women, 2190 new cases of breast cancer incidence and 410 deaths are expected in men in 2012 [1]. The current treatment modalities have severe adverse effects including endometrial cancer, cardiotoxicity, leukemia, blood clots, nausea, and vomiting, [1]. Hence, chemoprevention of cancer could be a better strategy.

The idea of cancer chemoprevention has received more attention since the promotion stage of breast cancer generally is a lengthy process that could be reversible [2]. Many natural products are under study to prevent cancer including breast cancer. One among those is auraptene from citrus fruits [3]. Previous studies with auraptene showed its chemopreventive effects in rodent models of many cancers including colon
[4-7], esophagus [8], tongue [9], liver [10, 11], and skin [3]. Our study demonstrated the chemopreventive effects of auraptene against breast cancer [12]. These studies in human breast carcinoma cell lines, MDA-MB-231, and MCF-7 indicated the anti-proliferative effects of citrus auraptene. Subsequently an in vivo study demonstrated that dietary auraptene (500 ppm in the diet) decreased mammary carcinoma incidence and delayed median time to tumor in $N$-methylnitrosourea (MNU) treated rats. HPLC analysis of the mammary tissues from auraptene treated rats detected auraptene in the low $\mu \mathrm{M}$ concentrations at both 200 and 500 ppm dose [12].

Many mechanisms have been attributed to the chemopreventive effects of auraptene. They include inhibition of polyamine synthesis $[9,13]$, induction of detoxifying enzymes $[14,15]$, induction of apoptosis $[3,16]$, inhibition of metalloproteinase $[17,18]$, and inhibition of cholesterol esterification [19] to name a few. Recently, Medina and colleagues reported that auraptene modulated genes under the transcriptional control of estrogen [19]. In our in vivo 
Effect of auraptene on cell cycle at $8 \mathrm{~h}$ after IGF-1 treatment

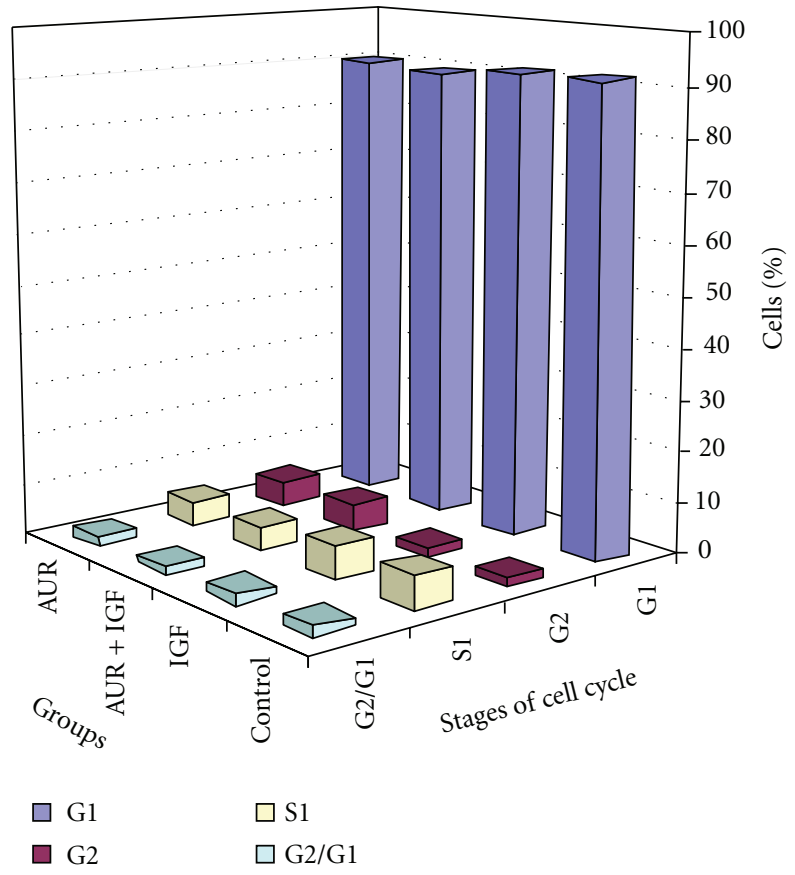

FIGURE 1: Cell cycle analysis of MCF-7 cells in the presence of IGF-1 at $8 \mathrm{~h}$. MCF-7 cells were serum starved for $24 \mathrm{~h}$. At $22 \mathrm{~h}$ after serum starvation the cells were treated with $10 \mu \mathrm{M}$ auraptene in $0.01 \%$ DMSO. At $24 \mathrm{~h}$ serum starvation, the cells were treated with IGF-1 $(10 \mathrm{ng} / \mathrm{mL})$. After $8 \mathrm{~h}$ of IGF-1 treatment, the cells were harvested and processed for cell cycle analysis by flow cytometry.

study, further analysis of the mammary tissues from rats showed that cyclin D1 protein was significantly reduced in auraptene $500 \mathrm{ppm}$ treated animals [12]. Cyclin D1 is a key regulatory protein in cell cycle (Figure 1 ). The D-type cyclins control the G1 to $\mathrm{S}$ transition during the cell cycle along with E-type cyclins [20]. In up to $50 \%$ of primary breast cancers the overexpression of cyclin D1 mRNA and protein has been observed [20]. Thus cyclin D1 is one of the most overexpressed oncogenes in human breast cancer. Cyclin D1 overexpression is predominantly found in estrogen receptor positive breast cancer, which is a major subtype of human breast cancer [20].

Since cyclin D1 protein expression was reduced in mammary tissues of auraptene treated rats, we explored the effect of auraptene on cell cycle in human breast carcinoma cell line MCF-7, which is positive for the estrogen receptor. The results shed more light on the inhibitory effects of auraptene on cell cycle machinery.

\section{Materials and Methods}

2.1. Chemicals. Auraptene was purchased from LKT Laboratories Inc. (St. Paul, MN). Recombinant human IGF-1 was obtained from Peprotech Inc. (Rocky Hill, NJ).
2.2. Cell Culture. Human MCF-7 mammary adenocarcinoma cells were purchased from ATCC (Manassas,VA). The cells were grown in sterile humidified chamber maintained at $37^{\circ} \mathrm{C}$ and $5 \% \mathrm{CO}_{2}: 95 \%$ air. The MCF-7 cells were grown and propagated in complete DMEM medium.

2.3. Cell Cycle Analysis. The effect of auraptene on cell cycle was studied in MCF-7 cells. Briefly, $1 \times 10^{6}$ cells were plated in eight $10 \mathrm{~cm}$ dishes, with duplicate dishes in each group. There were four groups: control (DMSO), IGF-1 only, IGF-1 + auraptene, and auraptene only. The cells were serum starved on the next day of plating. After $22 \mathrm{~h}$ of serum starvation, the IGF-1 + auraptene and auraptene only groups were pretreated with $10 \mu \mathrm{M}$ of auraptene in DMSO $(0.1 \%$ $\mathrm{v} / \mathrm{v})$. The control and IGF-1 only groups received vehicle. At $24 \mathrm{~h}$ of serum starvation, the IGF-1 only and IGF-1 + auraptene groups received $10 \mathrm{ng} / \mathrm{mL}$ of IGF- 1 . After $8 \mathrm{~h}$ and $24 \mathrm{~h}$ of IGF-1 treatment, the cells were harvested and centrifuged and the supernatant was removed. The pellets were then washed twice with ice cold PBS. The cells were counted and then fixed with ice cold $70 \%$ ethanol and kept at $4^{\circ}$ overnight. The next day, the cells were centrifuged, the ethanol was removed and the cells were washed twice with ice cold PBS. Then, to each tube $500 \mu \mathrm{L}$ of the propidium iodide solution mix was added (propidium iodide solution mix was prepared by adding $10 \mu \mathrm{L}$ of Triton-X $100(10 \%$ stock), $200 \mu \mathrm{L}$ of $10 \mathrm{mg} / \mathrm{mL}$ RNAse solution, and $400 \mu \mathrm{L}$ of $0.5 \mathrm{mg} / \mathrm{mL}$ propidium iodide solution to $10 \mathrm{~mL} \mathrm{PBS}$ ) and incubated for 30 minutes at room temperature, followed by cell cycle analysis using flow cytometry. The experiment was repeated 3 times. The flow cytometry was conducted using a BD LSRII (BD Biosciences) in the Research Core Facility at the LSUHSC-Shreveport. The instrument has a Coherent Sapphire laser for $488 \mathrm{~nm}$ excitation, a JDS Uniphase HeNe laser for $633 \mathrm{~nm}$ excitation, as well as a Coherent VioFlame for $450 \mathrm{~nm}$ excitation. The data were analyzed with FACS Diva (BD Biosciences) and ModFit LT software (Verify Software House).

2.4. $q R T-P C R$. A qRT-PCR array was done to analyze changes in gene expression of cell cycle related genes by auraptene. For this experiment, $1 \times 10^{5}$ cells were plated in eight $6 \mathrm{~cm}$ dishes, with duplicate dishes in each group. There were four groups: control, IGF-1 only, IGF-1 + auraptene, and auraptene only. The cells were serum starved on the next day of plating. At $22 \mathrm{~h}$ of serum starvation, the IGF-1 + auraptene and auraptene only groups were pretreated with $10 \mu \mathrm{M}$ of auraptene in DMSO $(0.1 \%, \mathrm{v} / \mathrm{v})$. The control and IGF-1 only groups received vehicle. At $24 \mathrm{~h}$ of serum starvation, the IGF-1 only and IGF-1 + auraptene groups received $10 \mathrm{ng} / \mathrm{mL}$ of IGF-1. After $8 \mathrm{~h}$ and $24 \mathrm{~h}$ of IGF-1 treatment, the cells were processed for RNA isolation as per the manufacturer's instructions (Qiagen, RNeasy minikit). The experiments were conducted in triplicate. The isolated RNA was treated with DNAse to remove any genomic contamination as per the manufacturer's suggested protocol (Promega). The DNA free RNA was then analyzed for quality on Agilent Analyzer. After confirming the quality of RNA, 
TABLE 1: Average percentage of cells at $8 \mathrm{~h}$ time point.

\begin{tabular}{lcccc}
\hline $\begin{array}{l}\text { Stage of cell } \\
\text { cycle }\end{array}$ & Control & IGF & IGF + auraptene & Auraptene \\
\hline G1 & $91.8 \pm 0.5$ & $91.3 \pm 0.2$ & $89.8 \pm 0.6$ & $90.2 \pm 0.94$ \\
G2 & $1.4 \pm 0.7$ & $1.9 \pm 0.9$ & $5.3 \pm 0.5$ & $4.8 \pm 0.8$ \\
S & $6.8 \pm 0.9$ & $6.7 \pm 1.2$ & $4.8 \pm 0.2$ & $4.9 \pm 0.3$ \\
G2/G1 & $2.0 \pm 0.1$ & $1.9 \pm 0.1$ & $1.8 \pm 0.0$ & $1.9 \pm 0.0$ \\
\hline
\end{tabular}

Figures represent means $\pm \operatorname{SEM}(n=3)$.

$1 \mu \mathrm{g}$ of RNA was converted to cDNA as per the directions from the manufacturer (Quanta Biosciences). The 1:10 dilution of cDNA was used for amplification with Perfecta SYBR Green Fast Mix for iQ (Quanta Biosciences) in the PCR Array (Human Cell Cycle Tox and Cancer 96 StellARray qPCR array, Lonza). The results from all experiments were analyzed using the Global Pattern Recognition Software from Bar Harbor BioTechnology, available on Lonza's website (http://array.lonza.com/stellarrays/). The experiment was repeated 3 times at $8 \mathrm{~h}$ and also at $24 \mathrm{~h}$ after IGF treatment. GPR fold changes were considered significant at $P \leq 0.05$.

2.5. Statistical Analysis. The analysis of cell cycle data was done by One-Way ANOVA followed by Tukey/Kramer post hoc test, $P<0.01$. The results from qRTPCR array studies were analyzed with the Global Pattern Recognition Software available on Lonza's website (http://array.lonza.com/stellarrays/), $P<0.05$.

\section{Results}

3.1. No Significant Change in the Percentage of Cells in the $S$ Phase after $8 \mathrm{~h}$ of IGF-1 Treatment. After $8 \mathrm{~h}$ of IGF-1 treatment, the harvested cells were run by flow cytometer to analyze the percentage of cells in the different phases of cell cycle (Figure 1 and Table 1). Most of the cells of the control group were in the G1 phase (92\%), and there were no significant differences in the percentages of cells in the G1 phase among the treatment groups. IGF-1 did not produce any significant increase in the percentage of cells in $S$ phase of the cell cycle at $8 \mathrm{~h}$ and no significant reduction in $\mathrm{S}$ phase was found in the auraptene treated cells. The percentage of cells in G2 in the control and the treatment groups also were almost the same. The effects of auraptene on cell cycle in the absence of IGF-1 was no different than the control group at $8 \mathrm{~h}$. Also, there were no apparent differences in the ratio of G2/G1.

3.2. Auraptene Significantly Reduced the Percentage of Cells in the S Phase after 24 h of IGF-1 Treatment. After $24 \mathrm{~h}$ of IGF-1 treatment, the harvested cells were run by flow cytometer to analyze the percentage of cells in the various phases of cell cycle. IGF-1 treatment resulted in a significantly decreased percentage of cells in the G1 phase compared to all the other groups from $87 \%$ in the control to $46 \%$ in the IGF- 1 treated group (Figure 2 and Table 2 ). There was a corresponding increase in the percentage of cells in S phase in the IGF-1
TABLE 2: Average percentage of cells at $24 \mathrm{~h}$ time point.

\begin{tabular}{lcccc}
\hline $\begin{array}{l}\text { Stage of cell } \\
\text { cycle }\end{array}$ & Control & IGF & IGF + auraptene & Auraptene \\
\hline G1 & $87.4 \pm 1.9$ & $42.6 \pm 0.4^{\mathrm{a}}$ & $87.4 \pm 2.0$ & $90.6 \pm 1.0$ \\
G2 & $1.9 \pm 1.5$ & $0.1 \pm 0.1$ & $3.2 \pm 1.1$ & $4.1 \pm 0.4$ \\
S & $10.7 \pm 0.5$ & $57.2 \pm 3.4^{\mathrm{a}}$ & $9.5 \pm 0.9$ & $5.4 \pm 0.7$ \\
G2/G1 & $2.0 \pm 0.0$ & $2.0 \pm 0.0$ & $2.0 \pm 0.0$ & $1.9 \pm 0.0$ \\
\hline
\end{tabular}

Figures represent means $\pm \operatorname{SEM}(n=3)$. ${ }^{\text {a Significantly different from }}$ control $P \leq 0.01$.

Effect of auraptene on cell cycle at $24 \mathrm{~h}$ after IGF-1 treatment

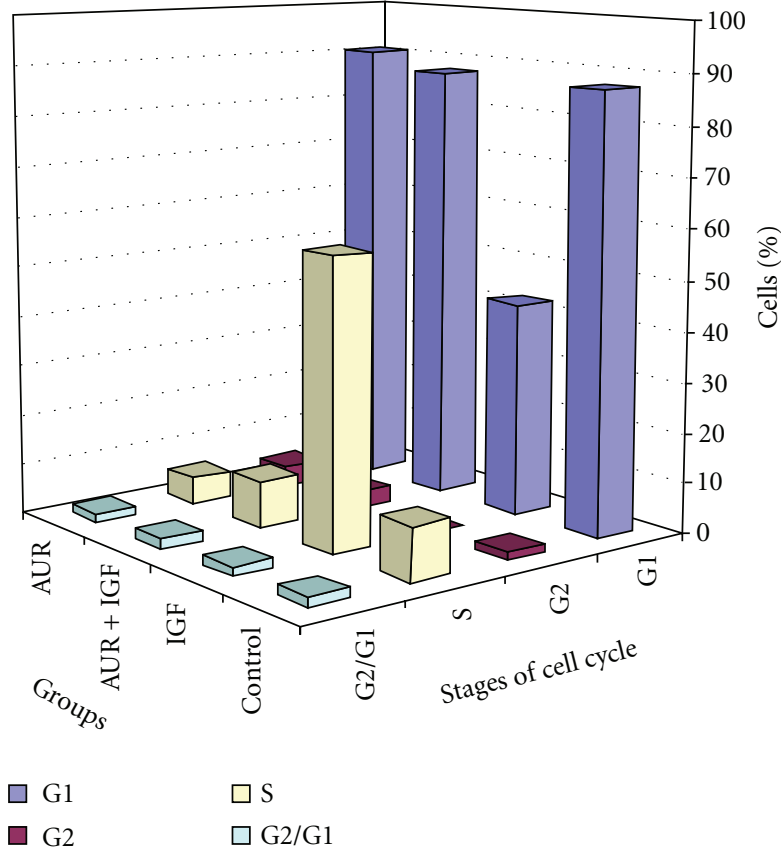

FIGURE 2: Cell cycle analysis of MCF-7 cells in the presence of IGF-1 at $24 \mathrm{~h}$. MCF-7 cells were serum starved for $24 \mathrm{~h}$. At $22 \mathrm{~h}$ after serum starvation the cells were treated with $10 \mu \mathrm{M}$ auraptene in $0.01 \%$ DMSO. After $24 \mathrm{~h}$ of serum starvation, the cells were treated with IGF-1 $(10 \mathrm{ng} / \mathrm{mL})$. After $24 \mathrm{~h}$ of IGF-1 treatment, the cells were harvested and processed for cell cycle analysis by flow cytometry.

treated group (from $10 \%$ in the control group to $57 \%$ in the IGF-1 treated group). Auraptene pretreatment significantly reduced the percentage of cells in S phase in the IGF-1 treated cells and appeared to restore the cells back to control levels of G1. The effects of auraptene on cell cycle in the absence of IGF-1 were no different than the control group at $24 \mathrm{~h}$. Also, there were no apparent differences in the ratio of G2/G1.

3.3. Auraptene Pretreatment in IGF-1 Treated MCF-7 Cells Significantly Modulated Several Genes Involved in Cell Cycle Regulation, Compared to IGF-1 Alone Treated Cells after $8 \mathrm{~h}$ of IGF-1 Treatment. In Table 3, the significant changes in the gene transcript level with auraptene pretreatment in IGF-1 treated cells when compared to the IGF-1 alone treated cells at $8 \mathrm{~h}$ time point are shown. There were significant changes in 9 genes, with 6 downregulated and 3 upregulated ones. The 
TABLE 3: Significant GPR fold change in the mRNA of target genes related to cell cycle in IGF-1 + auraptene treated cells compared to cells treated with IGF-1 alone at $8 \mathrm{~h}$.

\begin{tabular}{llr}
\hline Gene & Function & GPR fold change \\
\hline E2F1 & Act in the G1/S transition & -7.88 \\
CDC45L & DNA replication & -6.51 \\
E2F2 & Act in the G1/S transition & -9.81 \\
MCM3 & Integral part of the prereplication complex during cell cycle & -6.08 \\
MCM6 & Integral part of the prereplication complex during cell cycle & -3.71 \\
UHRF1 & Recruits histone deacetylase during cell cycle. Major role in G1/S transition & -17.01 \\
CDKN2B & Prevent activation of CDK by cyclin D1 & 5.83 \\
DDIT3 & Arresting cell cycle after DNA damage & 9.36 \\
JUN & Form AP-1 and promote cell proliferation & 3.65 \\
\hline
\end{tabular}

downregulated genes were E2F1 (E2F transcription factor 1), CDC45L (cell division cycle 45 homolog), E2F2 (E2F transcription factor 2), MCM3 (minichromosome maintenance complex component 3), MCM6 (minichromosome maintenance complex component 6), and UHRF1 (ubiquitin-like with $\mathrm{PHD}$ and ring finger domains 1). The upregulated genes include $C D K N 2 B$ (cyclin-dependent kinase inhibitor 2B), DDIT3 (DNA-damage-inducible transcript 3), and JUN (jun oncogene). The IGF-1 alone treatment resulted in the increased transcription of only 2 genes, MCM6 and ORC1L (origin recognition complex, subunit 1-like), which are key regulators of cell replication complex.

3.4. Auraptene Pretreatment in IGF-1 Treated MCF-7 Cells Significantly Modulated Several Genes Involved in Cell Cycle Regulation, Compared to IGF-1 Alone Treated Cells after $24 \mathrm{~h}$ of IGF-1 Treatment. In Table 4, the significant changes in the gene transcript level with auraptene pretreatment in IGF-1 treated cells when compared to the IGF-1 alone treated cells at $24 \mathrm{~h}$ time point are shown. There were 14 genes that changed significantly. Ten genes were downregulated while 4 were upregulated. The downregulated genes were CDC45L (cell division cycle 45 homolog), CDC2 (cyclindependent kinase 1), CCNA2 (cyclin A2), KIF20B (kinesin family member 20B), CHEK1 (CHK1 checkpoint homolog), CDKN2C (cyclin-dependent kinase inhibitor 2C), CHEK2 (CHK2 checkpoint homolog), E2F1 (E2F transcription factor 1), UHRF1 (ubiquitin-like with PHD and ring finger domains 1), and CCNB2 (cyclin B2). The upregulated genes were DDIT3 (DNA-damage-inducible transcript 3), CDKN2B (cyclin-dependent kinase inhibitor 2B), GADD45A (growth arrest and DNA-damage-inducible, alpha), and DUSP1 (dual specificity phosphatase 1). E2F1, CDC45L, UHRF1, DDIT3, and CDKN2B were modulated at both $8 \mathrm{~h}$ and $24 \mathrm{~h}$.

\section{Discussion}

Here, the effects of auraptene on cell cycle and genes involved in the mammalian cell cycle have been described. This study was conducted after finding the inhibitory effect of auraptene on cyclin D1 protein expression in MCF-7 cells and rat mammary tumors. We previously showed that in MCF-7 cells $10 \mu \mathrm{M}$ auraptene reduced the cyclin D1 protein expression by about $40 \%$ after treating with IGF-1. In the subsequent animal study, auraptene at $500 \mathrm{ppm}$ dose in the diet significantly delayed the time to tumor compared to the MNU only group. The rat mammary tumors from auraptene 500 ppm group showed significant reduction in cyclin D1 protein expression [12]. Since cyclin D1 is a key protein that regulates G1/S transition in cell cycle [21], we hypothesized that auraptene will inhibit the progression of cell cycle. To our knowledge this is the first study to show a detailing of the effect of auraptene on IGF-1 induced stimulation of the cell cycle in a breast cancer cell line. Auraptene significantly reduced the percentage of cells in the $S$ phase at the $24 \mathrm{~h}$ time point. This effect was not observed at the $8 \mathrm{~h}$ time point, even though auraptene reduced cyclin D1 expression after $8 \mathrm{~h}$ in MCF-7 cells [12]. This might be due to the fact that inhibition of cyclin D1 is an earlier event, which later resulted in slowing down the progression of cell cycle. The mitogenic activity of IGF-1 was not seen at $8 \mathrm{~h}$, since there was no significant increase in the percentage of cells in the $S$ phase compared to the control cells. At $24 \mathrm{~h}$, a significant increase in the percentage of cells in the $S$ phase was observed. Correspondingly, there was a significant reduction in the percentage of cells in the G1 phase of IGF-1 only group. IGF-1 was used to stimulate the serum starved MCF-7 cells because IGF-1 has been shown to be in high concentration in rats treated with MNU [22], which is the same carcinogen we used in our rat study with auraptene [12]. Elevated levels of IGF-1 are also found in human breast cancer patients [23]. Obese women, who are at higher risk to get breast cancer postmenopausally, also have higher IGF-1 blood levels [24]. It might be interesting to see the effect of auraptene in presence of other mitogens.

In the current study, after auraptene pretreatment, we saw a time dependent change in the transcriptional machinery associated with cell cycle along with the inhibition of $\mathrm{S}$ phase. At $8 \mathrm{~h}$ time point, significant changes were observed in 9 genes and at $24 \mathrm{~h}$ in 14 genes. Among these genes, 4 genes had a greater fold of change at the latter time point. Therefore, from the fact that these genes are still transcriptionally reduced, it is concluded that the effect of auraptene on cell cycle could persist for at least 
TABLE 4: Significant GPR fold change in the mRNA of target genes related to cell cycle in IGF-1 + auraptene treated cells compared to cells treated with IGF-1 alone at $24 \mathrm{~h}$ (the genes significantly changed at both $8 \mathrm{~h}$ and $24 \mathrm{~h}$ time points are shown in bold).

\begin{tabular}{llc}
\hline Gene & Function & GPR fold change \\
\hline CDC45L & DNA replication & $-\mathbf{2 0 . 7 1}$ \\
CDC2 & Promotes G1/S transition & -38.29 \\
CCNA2 & Promotes cell cycle in the mitosis phase & -20.25 \\
KIF20B & Involved in the cytokinesis, the final phase of cell division & -32.48 \\
CHEK1 & Inhibits cell division & -9.01 \\
CDKN2C & Inhibits G1/S entry & -8.98 \\
CHEK2 & Cell cycle arrest. Activated in response to DNA damage. \\
E2F1 & Act in the G1/S transition & -10.83 \\
CCNB2 & Promotes cell division, active in G2/M phase & $-\mathbf{1 0 . 4 4}$ \\
UHRF1 & Recruits histone deacetylase during cell cycle. Major role in G1/S transition & -6.81 \\
DDIT3 & Arresting cell cycle after DNA damage & $-\mathbf{4 5 . 8 5}$ \\
CDKN2B & Prevent activation of CDK by cyclin D1 & $\mathbf{5 3 . 2 2}$ \\
GADD45A & Participates in arresting cell cycle after DNA damage & $\mathbf{6 . 1 4}$ \\
DUSP1 & Inactivates MAPK phosphorylation and inhibits proliferation & 10.16 \\
\hline
\end{tabular}

$24 \mathrm{~h}$. This could be the effect of parent compound or its active metabolites. The major metabolites of auraptene are umbelliferone and 7-ethoxycoumarin which also have been shown to possess chemopreventive effects [25].

The cyclin D1 gene was also one of the cell cycle genes whose changes were analyzed along with the other mentioned genes. We found that there was no significant change in the mRNA level of cyclin D1 in the cells treated with IGF-1 and auraptene (data not shown). Therefore, auraptene's effect on cyclin D1 could be posttranscriptional. Previous studies have shown that auraptene changed the protein levels of COX-2, iNOS, and pro-MMP-7 without changing their transcript levels $[18,26]$. In the human colorectal adenocarcinoma cell line HT-29, auraptene disrupted the translation of proMMP-7 protein synthesis by decreasing phosphorylation levels of $4 \mathrm{E}$ binding protein (4EBP) 1 and eukaryotic translation initiation factor (eIF)4B [18]. These data along with our results point to the fact that auraptene might be acting on the translational machinery of the cells.

Many genes that were modulated by auraptene have been shown to be relevant in many cancers. Most of the prostate and breast-cancer cell lines studied had reduced copy number of $C D K N 2 B$ gene [27], whereas in this study we found it upregulated with time after auraptene pretreatment. CDKN2B, an inhibitor of cyclin D1 induced cell cycle progression from G1 phase to $S$ phase, could contribute to the auraptene induced inhibition of cell cycle at G1 phase. CDKN2B was found to be increased by auraptene to almost similar extent at both $8 \mathrm{~h}$ and $24 \mathrm{~h}$ whereas the $\mathrm{S}$ phase inhibition was evident at $24 \mathrm{~h}$. This might be because regulation of $C D K N 2 B$ might be an earlier event that leads to further inhibition of cell cycle machinery at a later time point. The E2F freed from $\mathrm{pRB}$ by the cyclin D-CDK complex upregulates the transcription of genes involved in DNAreplication-MCM components, cdc6, and cyclin E [28]. The E2F genes are deregulated in many cancers by various mechanisms like overexpression of cyclin D1, loss of pRB, and expression of human papillomavirus (HPV) oncoprotein E7, to name a few [29]. CDC45 protein has been shown to be overexpressed in various human cancer cell lines, including MCF-7 [30]. UHRF1 is under the transcriptional control of E2F1 transcription factor [31]. UHRF1 has been shown to be upregulated in cancer of breast, prostate, pancreas, and so forth [31-33]. Thus at the earlier time point in this study, the downregulation of cyclin D1 could have resulted in the reduced transcription of E2Fs and their downstream targets like UHRF1 and MCM complexes resulting in the initiation of cell cycle arrest. JUN or c-Jun is a component of the composite transcription factor activating protein-1 (AP-1) and promotes cell proliferation [34, 35]. C-Jun is overexpressed in many human cancers and contributes to the invasiveness of human breast cancer cells [36]. In this study, we found JUN upregulated at $8 \mathrm{~h}$ time point only and not at $24 \mathrm{~h}$ time point that corresponds with the inhibition of S phase at $24 \mathrm{~h}$ by auraptene.

At the later time point auraptene pretreatment resulted in greater change in the gene expression of CDC45L, E2F1, UHRF1, DDIT3, and CDK2NB. The other genes modulated by auraptene also have been shown to be deregulated in many cancers. The DUSP1 transcript was dramatically decreased in colorectal cancer compared to normal cells $[37,38]$. $\mathrm{Li}$ and colleagues [38] showed that DUSP1 (MKP1) is a transcriptional target of p53 that inhibits MAPK pathway activation of cell cycle progression. In estrogen dependent breast cancer cells, estrogen was shown to increase the expression of cyclin B2 protein and promote cell proliferation [39]. CHEK1 overexpression has been seen in human colorectal cancer correlated with advanced tumor and poor prognosis [40]. CDKN2C is the gene that codes for the cyclin dependent kinase 4 inhibitor $\mathrm{C}$ or p18. Mutation in p18 has been found in some human breast tumors [41]. Similar to $C D K N 2 B, C D K N 2 C$ also inhibits cyclin induced $\mathrm{G} 1 / \mathrm{S}$ transition. Therefore the increased expression of CDKN2C might also be playing an important role in auraptene induced inhibition of cell cycle at G1 phase. However these 2 genes, having similar functions, are modulated differently 
by auraptene at $8 \mathrm{~h}$ and $24 \mathrm{~h}$. KIF20B is a member of the kinesin-6 family [42]. It is involved in cytokinesis [43]. Several members of the KIF family are upregulated in cancer. In lung cancer, the KIF4A gene was observed to be highly transactivated [44]. In the majority of glioma cell lines, 3 genes were overexpressed: KIF1C, KIF3C, and KIF21B [45]. KIF20B was found to be highly expressed in a majority of human invasive bladder cancers [46]. Thus the overall gene changes brought about by auraptene pretreatment inhibited the progression of cell cycle at the G1 phase in MCF-7 cells.

A few of the above-mentioned genes have been identified as targets of anticancer therapy. CDC2 is also known as CDK1. CDK modulators have been investigated in clinical trials against many cancers. Flavopiridol, a CDK1 and CDK2 inhibitor [47], is being clinically tested against gastric cancer, leukemia, and head and neck cancer [48]. Another CDK1 modulator currently in clinical trials is UCN-01 [49]. In a cell cycle gene array experiment in MCF-10F cells with etodolac, a COX-2 inhibitor, CCNA2 was one of the prominently altered genes [50]. In PC-3 prostate cancer cells, luteolin reduced the transcription of $C C N A 2$ along with that of other cell cycle genes and inhibited the proliferation of those cells [51]. Since auraptene modulated these genes, further analysis of its potential as a dietary chemopreventive agent needs to be carried out.

\section{Conclusion}

Here we investigated the role of auraptene on cell cycle progression of human breast carcinoma cell line, MCF-7, and we showed the inhibitory effect of auraptene on cell cycle in MCF-7. Auraptene significantly reduced the percentage of cells undergoing $\mathrm{S}$ phase after $24 \mathrm{~h}$ of IGF-1 treatment. There were several genes involved in cell cycle that were significantly modulated with auraptene pretreatment. More genes were modulated at $24 \mathrm{~h}$ time point and correspondingly auraptene pretreatment showed inhibition of the cell cycle progression. Overall, auraptene pretreatment produced significant increase in the mRNA level of genes known to be upregulated prior to cell cycle arrest and apoptosis. There was a significant decrease in the mRNA level of genes that promote G1/S transition and DNA replication. Further studies on the effect of auraptene on cell cycle progression and protein translational machinery in different breast cancer cell lines will provide us with more information about its chemopreventive properties. However, the results obtained with auraptene in MCF-7 cells throw more light on cell cycle inhibition as one of its chemopreventive mechanisms. Since breast cancer is a multifactorial disease, a combination of drugs should be tried for chemoprevention studies and auraptene could be one of the candidates.

\section{Conflict of Interests}

The authors do not have any conflict of interests with the contents of this paper.

\section{Acknowledgment}

The authors received support from the Feist-Weiller Cancer Center, Department of Pharmacology, Toxicology \& Neuroscience. This paper was also supported, in part, by a National Cancer Institute Grant 1K22CA102005-01A2 (HKH). The content is solely the responsibility of the authors and does not necessarily represent the official views of the National Cancer Institute or the National Institutes of Health.

\section{References}

[1] American Cancer Society, Cancer Facts and Figures 2012, American Cancer Society, Atlanta, Ga, USA, 2012.

[2] Y. J. Surh, "Cancer chemoprevention with dietary phytochemicals," Nature Reviews Cancer, vol. 3, no. 10, pp. 768-780, 2003.

[3] A. Murakami, W. Kuki, Y. Takahashi et al., "Auraptene, a citrus coumarin, inhibits 12-O-tetradecanoylphorbol-13acetate-induced tumor promotion in ICR mouse skin, possibly through suppression of superoxide generation in leukocytes," Japanese Journal of Cancer Research, vol. 88, no. 5, pp. 443-452, 1997.

[4] T. Tanaka, Y. Yasui, R. Ishigamori-Suzuki, and T. Oyama, "Citrus compounds inhibit inflammation- and obesity-related colon carcinogenesis in mice," Nutrition and Cancer, vol. 60, supplement 1, pp. 70-80, 2008.

[5] K. Hayashi, R. Suzuki, S. Miyamoto et al., "Citrus auraptene suppresses azoxymethane-induced colonic preneoplastic lesions in C57BL/KsJ-db/db mice," Nutrition and Cancer, vol. 58, no. 1 , pp. 75-84, 2007.

[6] H. Kohno, R. Suzuki, M. Curini et al., "Dietary administration with prenyloxycoumarins, auraptene and collinin, inhibits colitis-related colon carcinogenesis in mice," International Journal of Cancer, vol. 118, no. 12, pp. 2936-2942, 2006.

[7] T. Tanaka, K. Kawabata, M. Kakumoto et al., "Citrus auraptene inhibits chemically induced colonic aberrant crypt foci in male F344 rats," Carcinogenesis, vol. 18, no. 11, pp. 2155-2161, 1997.

[8] K. Kawabata, T. Tanaka, T. Yamamoto et al., "Suppression of N-nitrosomethylbenzylamine-induced rat esophageal tumorigenesis by dietary feeding of auraptene," Journal of Experimental and Clinical Cancer Research, vol. 19, no. 1, pp. 45-52, 2000.

[9] T. Tanaka, K. Kawabata, M. Kakumoto et al., "Citrus auraptene exerts dose-dependent chemopreventive activity in rat large bowel tumorigenesis: the inhibition correlates with suppression of cell proliferation and lipid peroxidation and with induction of phase II drug- metabolizing enzymes," Cancer Research, vol. 58, no. 12, pp. 2550-2556, 1998.

[10] K. Sakata, A. Hara, Y. Hirose et al., "Dietary supplementation of the citrus antioxidant auraptene inhibits N,Ndiethylnitrosamine-induced rat hepatocarcinogenesis," Oncology, vol. 66, no. 3, pp. 244-252, 2004.

[11] A. Hara, K. Sakata, Y. Yamada et al., "Suppression of betacatenin mutation by dietary exposure of auraptene, a citrus antioxidant, in N,N-diethylnitrosamine-induced hepatocellular carcinomas in rats," Oncology Reports, vol. 14, no. 2, pp. 345-351, 2005.

[12] P. Krishnan, K. J. Yan, D. Windler et al., "Citrus auraptene suppresses cyclin D1 and significantly delays N-methyl nitrosourea induced mammary carcinogenesis in female Sprague-Dawley rats," BMC Cancer, vol. 9, p. 259, 2009.

[13] T. Tanaka, K. Kawabata, M. Kakumoto et al., "Chemoprevention of 4-nitroquinoline 1-oxide-induced oral carcinogenesis 
by citrus auraptene in rats," Carcinogenesis, vol. 19, no. 3, pp. 425-431, 1998.

[14] M. Prince, Y. Li, A. Childers, K. Itoh, M. Yamamoto, and H. E. Kleiner, "Comparison of citrus coumarins on carcinogendetoxifying enzymes in Nrf2 knockout mice," Toxicology Letters, vol. 185, no. 3, pp. 180-186, 2009.

[15] Q. Zheng, Y. Hirose, N. Yoshimi et al., "Further investigation of the modifying effect of various chemopreventive agents on apoptosis and cell proliferation in human colon cancer cells," Journal of Cancer Research and Clinical Oncology, vol. 128, no. 10, pp. 539-546, 2002.

[16] D. Y. Jun, J. S. Kim, H. S. Park et al., "Apoptogenic activity of auraptene of Zanthoxylum schinifolium toward human acute leukemia Jurkat $\mathrm{T}$ cells is associated with ER stress-mediated caspase- 8 activation that stimulates mitochondria-dependent or -independent caspase cascade," Carcinogenesis, vol. 28, no. 6, pp. 1303-1313, 2007.

[17] K. Kawabata, A. Murakami, and H. Ohigashi, "Auraptene decreases the activity of matrix metalloproteinases in dextran sulfate sodium-induced ulcerative colitis in ICR mice," Bioscience, Biotechnology and Biochemistry, vol. 70, no. 12, pp. 3062-3065, 2006.

[18] K. Kawabata, A. Murakami, and H. Ohigashi, "Citrus auraptene targets translation of MMP-7 (matrilysin) via ERK1/2-dependent and mTOR-independent mechanism," FEBS Letters, vol. 580, no. 22, pp. 5288-5294, 2006.

[19] P. de Medina, S. Genovese, M. R. Paillasse et al., "Auraptene is an inhibitor of cholesterol esterification and a modulator of estrogen receptors," Molecular Pharmacology, vol. 78, no. 5, pp. 827-836, 2010.

[20] R. L. Sutherland and E. A. Musgrove, "Cyclins and breast cancer," Journal of Mammary Gland Biology and Neoplasia, vol. 9, no. 1, pp. 95-104, 2004.

[21] C. J. Sherr and J. M. Roberts, "CDK inhibitors: positive and negative regulators of G1-phase progression," Genes and Development, vol. 13, no. 12, pp. 1501-1512, 1999.

[22] C. Cocca, A. Gutiérrez, M. Núñez et al., "Suppression of mammary gland tumorigenesis in diabetic rats," Cancer Detection and Prevention, vol. 27, no. 1, pp. 37-46, 2003.

[23] S. E. Hankinson, W. C. Willett, G. A. Colditz et al., "Circulating concentrations of insulin-like growth factor-I and risk of breast cancer," Lancet, vol. 351, no. 9113, pp. 1393-1396, 1998.

[24] G. D. Stephenson and D. P. Rose, "Breast cancer and obesity: an update," Nutrition and Cancer, vol. 45, no. 1, pp. 1-16, 2003.

[25] A. Murakami, K. Wada, N. Ueda et al., "In vitro absorption and metabolism of a citrus chemopreventive agent, auraptene, and its modifying effects on xenobiotic enzyme activities in mouse livers," Nutrition and Cancer, vol. 36, no. 2, pp. 191199, 2000.

[26] A. Murakami and H. Ohigashi, "Cancer-preventive antioxidants that attenuate free radical generation by inflammatory cells," Biological Chemistry, vol. 387, no. 4, pp. 387-392, 2006.

[27] M. Yamamoto, R. Metoki, and F. Yamamoto, "Systematic multiplex polymerase chain reaction and reverse transcriptionpolymerase chain reaction analyses of changes in copy number and expression of proto-oncogenes tumor suppressor genes in cancer tissues and cell lines," Electrophoresis, vol. 25, no. 20, pp. 3349-3356, 2004.

[28] S. Polager and D. Ginsberg, "E2F-at the crossroads of life and death," Trends in Cell Biology, vol. 18, no. 11, pp. 528-535, 2008.
[29] C. J. Sherr and F. McCormick, "The RB and p53 pathways in cancer," Cancer Cell, vol. 2, no. 2, pp. 103-112, 2002.

[30] S. Pollok, C. Bauerschmidt, J. Sänger, H. P. Nasheuer, and F. Grosse, "Human Cdc45 is a proliferation-associated antigen," FEBS Journal, vol. 274, no. 14, pp. 3669-3684, 2007.

[31] M. Unoki, T. Nishidate, and Y. Nakamura, "ICBP90, an E2F-1 target, recruits HDAC1 and binds to methyl-CpG through its SRA domain," Oncogene, vol. 23, no. 46, pp. 7601-7610, 2004.

[32] M. Mousli, R. Hopfner, A. Q. Abbady et al., "ICBP90 belongs to a new family of proteins with an expression that is deregulated in cancer cells," British Journal of Cancer, vol. 89, no. 1, pp. 120-127, 2003.

[33] M. Lorenzato, S. Caudroy, C. Bronner et al., "Cell cycle and/or proliferation markers: what is the best method to discriminate cervical high-grade lesions?" Human Pathology, vol. 36, no. 10, pp. 1101-1107, 2005.

[34] T. D. Halazonetis, K. Georgopoulos, M. E. Greenberg, and P. Leder, "c-Jun dimerizes with itself and with c-Fos, forming complexes of different DNA binding affinities," Cell, vol. 55, no. 5, pp. 917-924, 1988.

[35] P. Angel and M. Karin, "The role of Jun, Fos and the AP-1 complex in cell-proliferation and transformation," Biochimica et Biophysica Acta, vol. 1072, no. 2-3, pp. 129-157, 1991.

[36] J. Wang, I. Kuiatse, A. V. Lee, J. Pan, A. Giuliano, and X. Cui, "Sustained c-Jun-NH2-kinase activity promotes epithelialmesenchymal transition, invasion, and survival of breast cancer cells by regulating extracellular signal-regulated kinase activation," Molecular Cancer Research, vol. 8, no. 2, pp. 266277, 2010.

[37] L. Zhang, W. Zhou, V. E. Velculescu et al., "Gene expression profiles in normal and cancer cells,” Science, vol. 276, no. 5316, pp. 1268-1272, 1997.

[38] M. Li, J. Y. Zhou, Y. Ge, L. H. Matherly, and G. S. Wu, “The phosphatase MKP1 is a transcriptional target of p53 involved in cell cycle regulation," Journal of Biological Chemistry, vol. 278, no. 42, pp. 41059-41068, 2003.

[39] Q. Felty, K. P. Singh, and D. Roy, "Estrogen-induced G1/S transition of G0-arrested estrogen-dependent breast cancer cells is regulated by mitochondrial oxidant signaling," Oncogene, vol. 24, no. 31, pp. 4883-4893, 2005.

[40] H. Gali-Muhtasib, D. Kuester, C. Mawrin et al., "Thymoquinone triggers inactivation of the stress response pathway sensor CHEK1 and contributes to apoptosis in colorectal cancer cells," Cancer Research, vol. 68, no. 14, pp. 5609-5618, 2008.

[41] A. Blais, Y. Labrie, F. Pouliot, Y. Lachance, and C. Labrie, "Structure of the gene encoding the human cyclin-dependent kinase inhibitor p18 and mutational analysis in breast cancer," Biochemical and Biophysical Research Communications, vol. 247, no. 1, pp. 146-153, 1998.

[42] H. Miki, Y. Okada, and N. Hirokawa, "Analysis of the kinesin superfamily: insights into structure and function," Trends in Cell Biology, vol. 15, no. 9, pp. 467-476, 2005.

[43] R. R. Adams, A. A. M. Tavares, A. Salzberg, H. J. Bellen, and D. M. Glover, "pavarotti encodes a kinesin-like protein required to organize the central spindle and contractile ring for cytokinesis," Genes and Development, vol. 12, no. 10, pp. 1483-1494, 1998.

[44] M. Taniwaki, A. Takano, N. Ishikawa et al., "Activation of KIF4A as a prognostic biomarker and therapeutic target for lung cancer," Clinical Cancer Research, vol. 13, no. 22, pp. 6624-6631, 2007.

[45] M. Harada, Y. Ishihara, K. Itoh, and R. Yamanaka, "Kinesin superfamily protein-derived peptides with the ability to 
induce glioma-reactive cytotoxic T lymphocytes in human leukocyte antigen-A24+ glioma patients," Oncology Reports, vol. 17, no. 3, pp. 629-636, 2007.

[46] M. Kanehira, T. Katagiri, A. Shimo et al., "Oncogenic role of MPHOSPH1, a cancer-testis antigen specific to human bladder cancer," Cancer Research, vol. 67, no. 7, pp. 3276-3285, 2007.

[47] G. Kaur, M. Stetler-Stevenson, S. Sebers et al., "Growth inhibition with reversible cell cycle arrest of carcinoma cells by flavone L86-8275," Journal of the National Cancer Institute, vol. 84, no. 22, pp. 1736-1740, 1992.

[48] A. M. Senderowicz, "Small-molecule cyclin-dependent kinase modulators," Oncogene, vol. 22, no. 43, pp. 6609-6620, 2003.

[49] Y. Dai and S. Grant, "Small molecule inhibitors targeting cyclin-dependent kinases as anticancer agents," Current Oncology Reports, vol. 6, no. 2, pp. 123-130, 2004.

[50] D. Roy, G. A. Arason, B. Chowdhury, A. Mitra, and G. M. Calaf, "Profiling of cell cycle genes of breast cells exposed to etodolac," Oncology Reports, vol. 23, no. 5, pp. 1383-1391, 2010.

[51] K. Shoulars, M. A. Rodriguez, T. Thompson, and B. M. Markaverich, "Regulation of cell cycle and RNA transcription genes identified by microarray analysis of PC-3 human prostate cancer cells treated with luteolin," Journal of Steroid Biochemistry and Molecular Biology, vol. 118, no. 1-2, pp. 4150, 2010. 


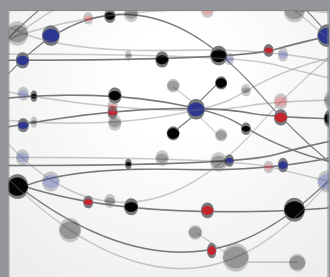

The Scientific World Journal
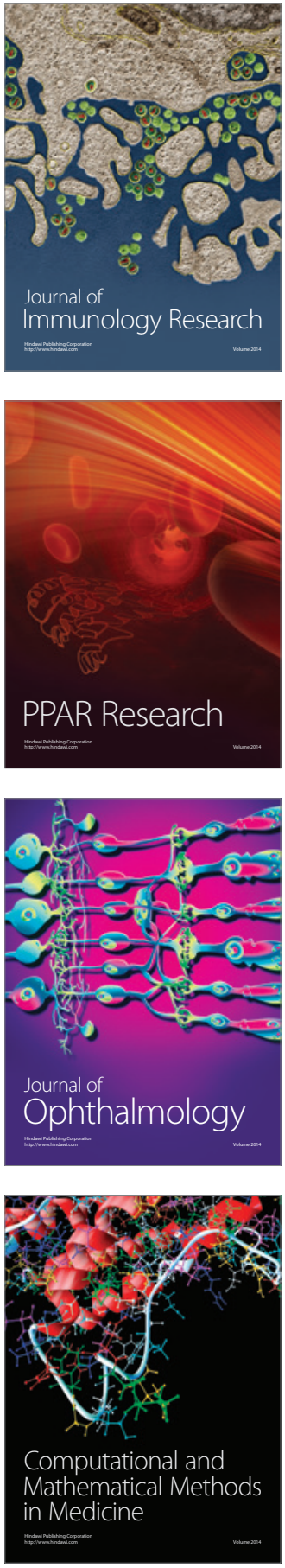

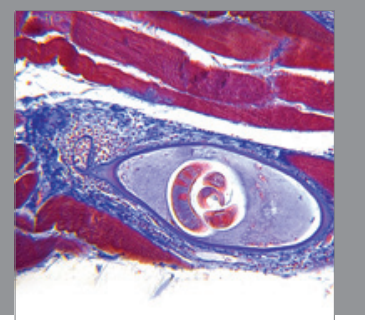

Gastroenterology

Research and Practice
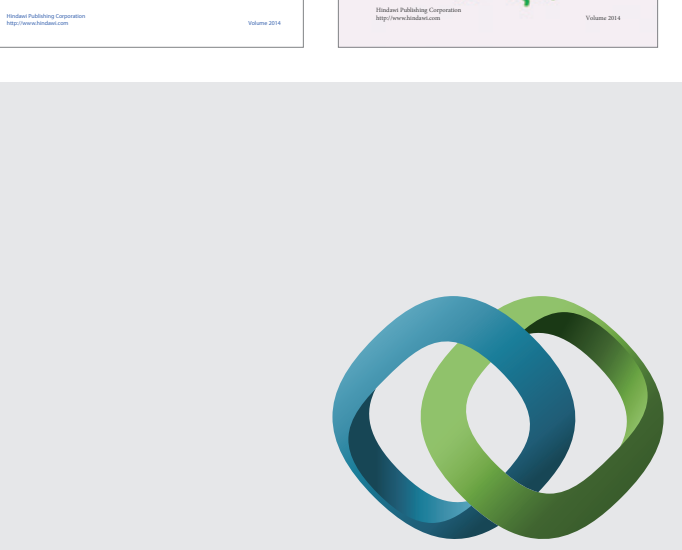

\section{Hindawi}

Submit your manuscripts at

http://www.hindawi.com
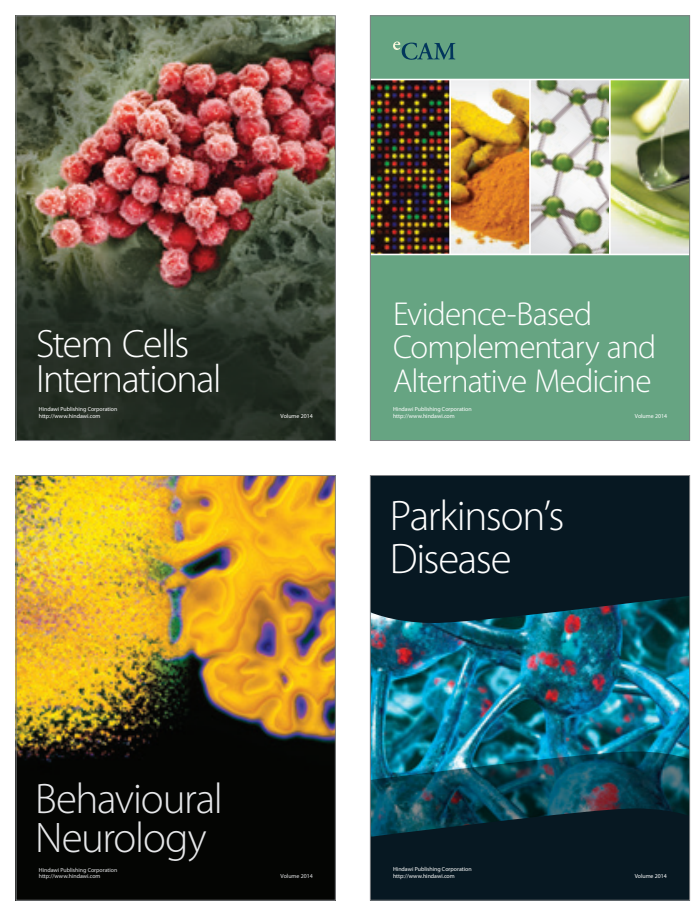

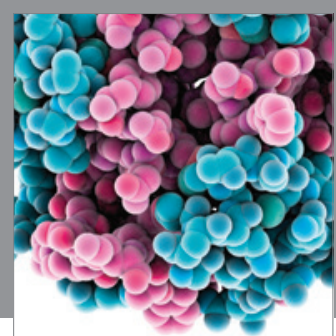

Journal of
Diabetes Research

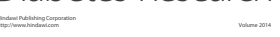

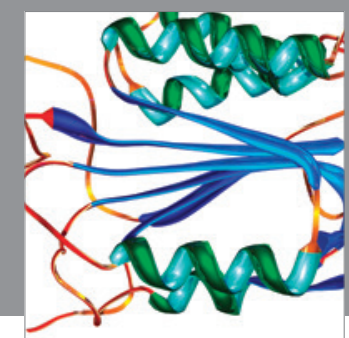

Disease Markers
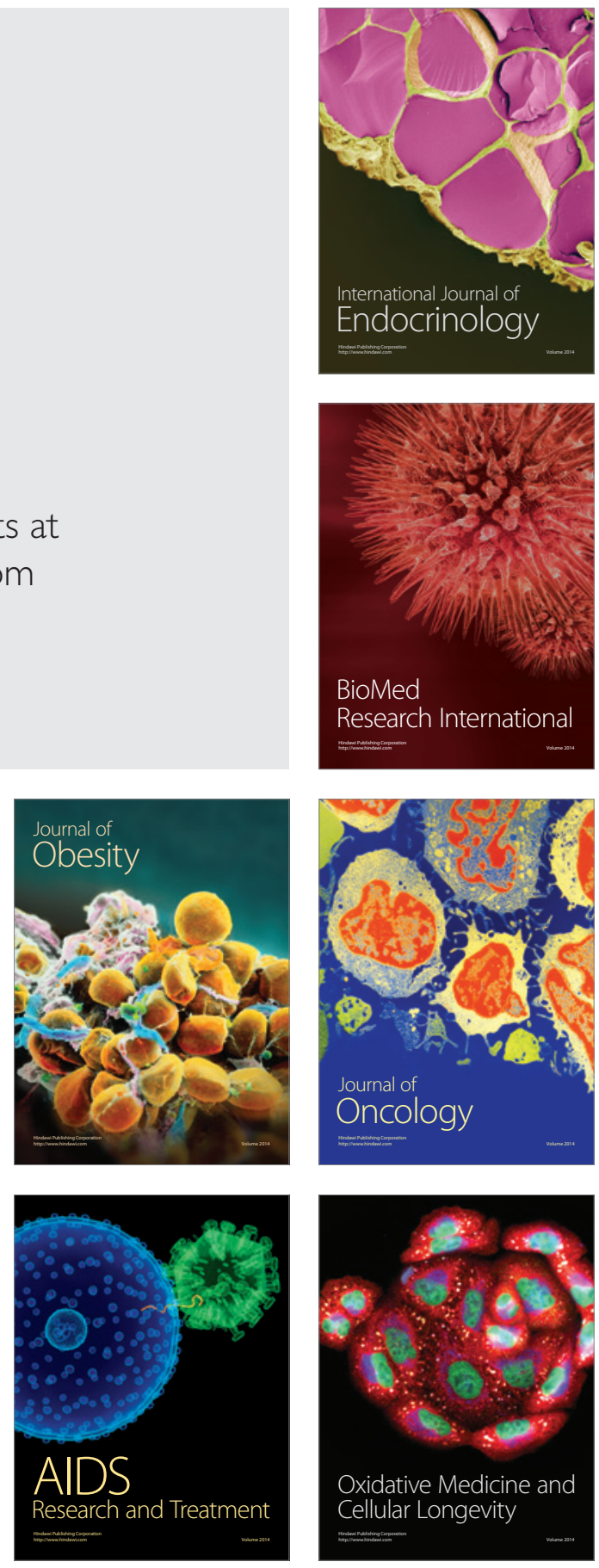